INPLASY

PROTOCOL

To cite: Li et al. Associations between hepatectomy and survival of gastric cancer patients with liver metastases: A network pooling analysis. Inplasy protocol 2020120009. doi:

10.37766/inplasy2020.12.0009

Received: 02 December 2020

Published: 02 December 2020

Corresponding author:

Min Sun

sunmin-0715@163.com

Author Affiliation:

Department of General

Surgery, Taihe Hospital, Hubei

University of Medicine, Shiyan

442000, China

Support: NSFC (81902498).

Review Stage at time of this submission: Data analysis.

Conflicts of interest:

None.

\section{Associations between hepatectomy and survival of gastric cancer patients with liver metastases: A network pooling analysis}

$\mathrm{Li}, \mathrm{T} 1$; Sun, $\mathrm{M}^{2}$.
Review question / Objective: Gastric cancer with liver metastases (GCLM) is considered a late stage disease. Systemic chemotherapy was recommended as standard cure, with a 5-year survival rate of less than $10 \%$. The current standard management of GCLM is systemic chemotherapy with supportive care. The application of hepatectomy combined with radical gastrectomy for GCLM is controversial. The role of liver resection for GCLM is gradually being considered.

INPLASY registration number: This protocol was registered with the International Platform of Registered Systematic Review and Meta-Analysis Protocols (INPLASY) on 02 December 2020 and was last updated on 02 December 2020 (registration number INPLASY2020120009).

\section{INTRODUCTION}

Review question / Objective: Gastric cancer with liver metastases (GCLM) is considered a late stage disease. Systemic chemotherapy was recommended as standard cure, with a 5-year survival rate of less than $10 \%$. The current standard management of GCLM is systemic chemotherapy with supportive care. The application of hepatectomy combined with radical gastrectomy for GCLM is controversial. The role of liver resection for GCLM is gradually being considered.

Rationale: According to the guidelines of The Committee of the Japan Gastric Cancer Association (JGCA) and National Comprehensive Cancer Network (NCCN), palliative management is recommended for 
stage IV gastric cancer, e.g. GCLM. In contrast, colorectal liver metastases are considered as suitable targets for radical surgery because they often present as liver-only metastatic disease, and R0 resection shows good prognostic outcomes, with a 5-year survival rate > $50 \%$. Retrospective studies have presented that the combination of hepatectomy and gastrectomy has visible survival outcome superiority. Compared to systemic chemotherapy, surgical treatment of hepatic metastases presents favorable prognosis. In the last two decades, along with the results of reported studies which demonstrated that radical surgery of primary gastric cancer and metastatic liver lesions had survival benefits, the Guidelines Committee of JGCA reconsidered the effect of surgical treatment in GCLM patients.

Condition being studied: Previous therapeutic options for GCLM were systemic chemotherapy (CT), gastrectomy plus chemotherapy (G), hepatic interventional therapy plus gastrectomy (IT), and hepatectomy plus gastrectomy (HG). There are no randomized controlled clinical trials for GCLM therapies. In the present literature, majority of the studies are retrospective studies, which were performed at a single center, with a limited number of patients. Although some studies have confirmed the superior therapeutic outcomes of HG, the clinical pathological characteristics of the involved patients reveal some selection bias, therefore, their results are difficult to accept. We performed a network meta-analysis to evaluate the survival benefits of surgical treatment and systemic chemotherapy in the treatment of GCLM.

\section{METHODS}

Search strategy: We retrieved literature published in between 1966 and December 1st, 2018 by searching PubMed, EMBASE, and Cochrane Library with the keywords (1) "stomach neoplasm" OR "gastric neoplasms" OR "cancer of stomach" OR "stomach cancers" OR "gastric cancer" AND (2) "liver metastases OR liver metastasis OR hepatic metastasis" AND (3) "operative surgical procedure" OR ablation OR liver resection OR hepatectomy OR gastrectomy OR chemotherapy OR "interventional therapy". We selected and evaluated all relevant studies and review articles about GCLM and inquired the authors for unpublished raw data. Searches were limited to English-language publications. In addition, the reference lists of the retrieved articles were examined for potential eligible studies.

Participant or population: Adults ( $\geq 18$ y) with gastric cancer with liver metastases, irrespective of gender, and race, irrespective of diabetes. The therapeutic options for gastric cancer with liver metastases were systemic chemotherapy (CT), gastrectomy plus chemotherapy (G), hepatic interventional therapy plus gastrectomy (IT), and hepatectomy plus gastrectomy (HG).

Intervention: The therapeutic options for gastric cancer with liver metastases were systemic chemotherapy (CT), gastrectomy plus chemotherapy (G), hepatic interventional therapy plus gastrectomy (IT), and hepatectomy plus gastrectomy (HG).

Comparator: The therapeutic options for gastric cancer with liver metastases were systemic chemotherapy (CT), gastrectomy plus chemotherapy (G), hepatic interventional therapy plus gastrectomy (IT), and hepatectomy plus gastrectomy (HG).

Study designs to be included: Case-control studies and cohort studies

Eligibility criteria: The inclusion criteria for the studies was: i. Systemic chemotherapy and surgical treatment; ii. Series of case control or cohort studies; iii. The number of patients were to be > 20; iv. Consists of available endpoints, such as 1-, 2- , 3- , and 5-year survival rates, median survival time, and postoperative complications. The exclusion criteria for the studies was: $i$. studies with insufficient data or no related endpoints; ii. Missing control group. 
Information sources: Two researchers independently extracted results from the enrolled articles in a standardized form. In addition, a third researcher was consulted in case there were disagreements. The information extracted from each study included the first author, country, year of publication, number of cases, treatment, sex, median or mean age of patients, study design, follow-up, median survival time.

Main outcome(s): 1) 1- year overall survival rates. 2) 2- year overall survival rates. 3) 3year overall survival rates. 4) 5-year overall survival rates.

\section{Additional outcome(s): None.}

Data management: Two researchers independently extracted results from the enrolled articles in a standardized form. In addition, a third researcher was consulted in case there were disagreements. The information extracted from each study included the first author, country, year of publication, number of cases, treatment, sex, median or mean age of patients, study design, follow-up, median survival time.

Quality assessment / Risk of bias analysis: We used the Newcastle-Ottawa Scale (NOS) to assess the quality of each included study. Scores $\geq 7$ were considered high quality. We used a "star system" for case-control studies.

Strategy of data synthesis: A pair-wise meta-analysis was performed by STATA 13.0 (Stata Corp, College Station, TX). 1-, 2-, 3- and 5-year overall survival rates were analyzed while relative risk (RR) with 95\% confidence intervals (Cls) was calculated by fixed-effects or random-effects model. Z test was performed to evaluate the significance of overall effect size. A network plot was then used to directly demonstrate the whole information of included studies. Depending on direct comparison and indirect comparison outcomes, we estimated the contribution of each direct treatment comparison in the whole network structure, which was presented in a contribution plot. The inconsistency factor (IF) was calculated to determine the possible inconsistency in network comparison. The $95 \%$ Cls of IF values close to zero or the $p$ value of $Z$ test higher than 0.05 demonstrated there being no statistically significant inconsistency. Summary effects and corresponding predictive intervals were used to conclude relative mean effects and impact of heterogeneity in the network forest plot. Finally, we calculated the surface under the cumulative ranking curve (SUCRA) of each treatment, which transformed the relative effects to the probability. The treatment was more valuable if the SUCRA value was higher. According to the estimated probability outcomes, the treatments were ranked. Small-study effects was adjusted by a model of network meta-regression, the variance of the log-odds ratios as covariation.

Subgroup analysis: None.

Sensibility analysis: None.

Language: Searches were limited to English-language publications.

Country(ies) involved: China.

Keywords: Gastric cancer; Liver metastasis; Hepatectomy; Interventional therapy; Network meta-analysis.

Contributions of each author:

Author 1 - Tian Li - The author drafted the manuscript.

Author 2 - Min Sun - The author participated in study design and performed data collection and data analysis. 